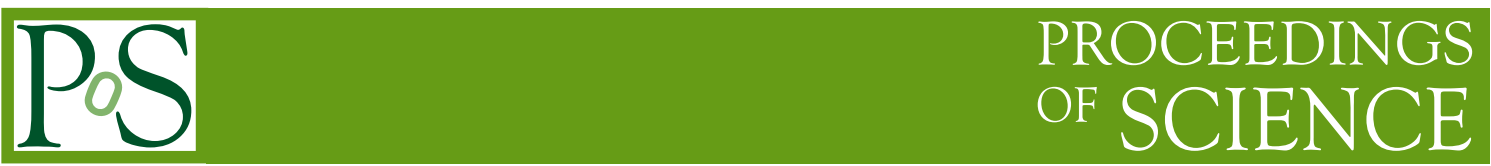

\title{
Bottom-up modelling of gamma-ray AGNs
}

\author{
Joni Tammi* \\ Aalto University Metsähovi Radio Observatory, P.O.Box 13000, FI-00076 Aalto, FINLAND \\ E-mail: joni.tammi@iki.fi
}

I discuss the role of radio observations and modelling of the low-frequency emission in active galactic nuclei (AGN). I emphasise the significance of the synchrotron spectrum in understanding the sources and modelling their gamma-ray emission. I discuss some of the AGN-specific parts of the Planck early-release data analysis published elsewhere [1]. The focus of the discussion is on modelling the blazar spectral energy distribution and gamma-ray variability using a "bottom-up" approach, from the point of view of the radiating particles, emphasising the role of low-energy emission in determining the parameters and conditions responsible for the high-energy emission.

The Extreme and Variable High Energy Sky - extremesky2011,

September 19-23, 2011

Chia Laguna (Cagliari), Italy

* Speaker. 


\section{Introduction}

Synchrotron emission is the first and the most straightforward observable signature of the particles and the environment that produce the high-energy spectra and variability, but only a part of the observations are typically used to constrain models. Typically only the IR-to-optical-to-UV part of the synchrotron component is compared to the observations and the radio flux is taken to originate from older components, in the jet, or be otherwise disconnected from the high-energy flaring. Even advanced models that pay close attention to the particles and the synchrotron spectrum (see [2,3] for examples), tend to focus on the optical-UV part. For the simple single-zone models concentrating only on the the fast high-energy variability and the upper end of the particle energy distribution this is understandable, but in going beyond the one-zone approach, the wealth of information in the radio-to-IR regime can provide significant insights into the shape of the particle spectra, cooling and acceleration processes, and the underlying jet (e.g. [4]).

Reviews of the current views of blazar spectral modelling can be found elsewhere (e.g. [5]), but one of the most heated topics currenly deal with the origin of the origin of the gamma-rays: where are they produced and by what mechanism? In the leptonic models the main alternatives are either the external inverse Compton (EC) in the innermost part of the AGN, or by the synchrotron self-Compton (SSC) farther down in the jet. Traditionally, the innermost jet has been the strongest candidate for the origin of high-energy photons, and the violent, extreme conditions near the black hole are the likely culprits for the extreme radiation. Recently observed high-energy cutoffs in some blazars have been suggested to be evidence of absorption due to photon-photon interactions within the BLR [6], or otherwise near the central engine [7], but, on the other hand, in other sources the cut-offs have been shown not to exist (e.g., [8,9]). Furthermore, statistical studies suggesting strong gamma-ray flares often happening after the onset of radio flares [10-12] and after the ejection of new VLBI components $[13,14]$ make a strong argument for some of the gamma-ray flaring farther away in the jet, parsecs away from the black hole [15-17].

Taking into account the emission from shock moving in the jet, regardless of the exact mechanism behind the high-energy peak, young shocks in the jet can also contribute to the IC spectrum, and this contribution needs to be taken into account in the model fits. In some sources the shocks can produce a part or all of the observed X- and gamma-ray emission through the SSC mechanism (e.g., [4, 3], and, as reported by [18], higher-order SSC might be enough to account for the gamma-rays in some cases. On the other hand, models including both SSC and EC from various seed photon sources suggest that the highest-energy gamma-rays could be dominated by the EC process (e.g., [2]). In particular, as mentioned above, the $\mathrm{GeV}$ break observed in 3C 454.3 [19], for example, appears to be best explained by EC models [6, 7].

Furthermore, in many cases there are multiple jet components contributing to the total SED at the same time. As a component ages it goes through various phases and emits a different spectrum in each phase [20,21], and by modelling the evolution of the components it is possible to trace the spectral variability of many sources in great detail from radio to optical (for example [4]). The possibility of contribution from multiple components needs to be taken into account when testing the models, and long-term multifrequency lightcurve and VLBI monitoring are needed to limit the number of free parameters. 


\section{The Planck early-release AGN spectra}

Although the Planck satellite is mainly focused on the cosmic microwave background, it also scans the "foreground" objects like active galaxies bright at the radio-to-mm frequencies. [1] published the spectral energy distributions of a hundred radio-bright northern AGNs from the first Planck all-sky survey, taken between August 2009 and June 2010 covering nine frequency bands between 30 and $857 \mathrm{GHz}$, together with simultaneous observations from collaborators across the electromagnetic spectrum. These early results mostly include one or two observing epochs depending on the source, and for many sources the published SED is the average of the two. The final release of the data will provide us with the complete SEDs for individual epochs for all observed sources, but already now a couple of interesting implications can be pointed out. In the following, all quantitative results are taken from [1] where they are discussed in detail. The power-law spectral indices $\alpha$ and $s$ are used for the photon spectrum $S_{v} \propto v^{\alpha}$ and the particle energy distribution $N(E) \propto E^{-s}$, correspondingly.

First of all, the Planck radio spectra are generally flatter than one might expect. At lower frequencies $(\leq 70 \mathrm{GHz})$ the spectral indices concentrate around $\alpha \approx 0$ and on higher frequencies ( $>70 \mathrm{GHz}$ ) the average index is $\alpha \approx-0.6$. For optically thin synchrotron spectrum the spectral index depends on the particle energy spectral index according to $\alpha_{\text {thin }}=(1-s) / 2$. Traditionally $s$ for the synchrotron-emitting particles is expected to lie between 2.2 and 2.5, corresponding to $\alpha$ between -0.6 and -0.75 - the majority of the high-frequency spectra are flatter than this.

The lower-frequency flatness is usually taken to be due to either an unresolved, optically thick core or a combination of aging synchrotron jet components moving to lower frequencies. Multiple individual spectra with different ages and self-absorption turnover peak frequencies are known to create a generally flat but bumpy spectrum [22]. It is likely that also here the low-frequency flatness in many sources is due to superposition of multiple synchrotron components, although in some cases the spectrum is so smooth that the multi-component explanation seems unlikely.

The higher-frequency spectral flatness seen in many sources is even more interesting. Even though many of the spectra have the "expected" optically thin spectral index between -0.7 and -1.2 (synchrotron losses steepen the spectrum by an additional $\Delta \alpha=-0.5$ ), most of them are harder that this. 15 sources even have a high-frequency spectrum flatter than -0.3 , and in many cases the high-frequency spectrum appears to be straight within the error bars. Even though the early release data are not sufficient enough to be definite, and while some individual sources do show evidence of several spectral components, [1] considered the multi-component explanation unlikely for the whole sample.

In addition to general statistical results, [1] also modelled a few example sources by fitting simple synchrotron spectra to the radio-to-optical spectra assuming radio outbursts corresponding to new shocks appearing in the jet. Long-term radio lightcurves were analysed to gain information regarding the past activity of the sources. In particular, they listed 10 sources that were observed only once (to exclude the flatness caused by averaging over multiple epochs) and whose highfrequency radio spectrum was flatter than $\alpha \gtrsim-0.5$. For seven of these they ruled out flatness due to multiple components and claimed the spectrum to be straight within the error bars. In these example cases the synchrotron spectrum could not be explained with standard acceleration scenario and particle spectra with $s \geq 2$. Instead, the observations are compatible with an electron index 
$s \approx 1.5$ and $\alpha_{\text {thin }}$ having flattest values around -0.2 and the steepest around -0.7 (presumably after the steepening due to synchrotron losses). Similar suggestions have been made already earlier by, e.g., [23-25].

Furthermore, in sources undergoing a major outburst a single dominating synchrotron component can fit the data from radio to optical. One example, 3C 454.3, was fitted using a numerical model (Tammi et al., in preparation), and the spectrum, obtained during the early stages of the strongest outburst observed for this source, shows two separate structures best modelled with jet emission at the lowest frequencies and a synchrotron spectrum from a strong shock-in-jet component dominating the sub-millimetre-to-optical spectrum. Similarly for those sources that had multiple outbursts before the Planck but no ongoing activity during the observation, the low-frequency spectrum could not be fitted with a single component, but required multiple synchrotron components, again in good agreement with expectations within the shock-in-jet scenario.

As said, the results are in general compatible with an assumption of a shock in the jet, and with an electron index $s \approx 1.5$. This is significantly harder than the $\sim 2.2-2.5$ associated with the standard first-order Fermi acceleration scenario, but as discussed in [1], it is possible to have harder spectra by making assumptions about the shock geometry or the particle scattering process, or when the turbulence effects across the shock are considered. The last alternative of these is an especially interesting one, because the combination of low matter density and relatively strong magnetic field can enhance the effective strength of the shock and lead to very hard particle spectra $[26,27$, and references therein].

Furthermore, in these conditions also the second-order Fermi acceleration can become effective and lead to very hard power-law spectra as well as enable high injection energies for the first-order shock acceleration on time-scales comparable to the fastest gamma-ray flares [28, 29]. Although there are only a few models that include the effects of the second-order mechanism and time-dependent particle acceleration, the attempts so far have been successful and encouraging $[3,30$, for example].

\section{Summary}

The synchrotron emission originating in the shocks in the jet is the primary signature of the relativistic particles that are responsible for the IC scattering regardless of the origin of the upscattered seed photons. Radio spectra and lightcurves covering also previous outbursts, together with detailed modelling of the synchrotron spectrum, offer information and tests that benefit also gamma-ray models through better understanding of the energetic particles and the radiation environment.

Recent Planck early results have increased motivation and need for developing multi-zone and multi-component models and working our way from the bottom up, from particle-level physics to the synchrotron spectrum and all the way to the observed EC and SSC variability. The early modelling attempts highlight the importance of accurate radio-to-submillimetre observations and synchrotron modelling even in blazar models concentrating on the highest energies. Based on the early release data of about one hundred northern AGNs over Planck frequencies and simultaneous multifrequency observations, the following points are the most relevant relevant in the scope of high-energy modelling [1]. 
In many sources the history of past activity can still be seen in the low-frequency radio spectra, which often show signs of multiple synchrotron components of different ages contributing to the total SED. On the other hand, in cases where the source shows ongoing millimetre-to-submillimetre flaring the radio-to-optical spectrum can be modelled with one dominating synchrotron component from the ongoing outburts. Different emission sites mean different sets of parameters, highlighting the unsuitability of one-zone models for accurate modelling of these sources. The number of components affecting the observations at a given frequency range can be estimated from long-term multifrequency lightcurves and VLBI monitoring.

Finally, the high-frequency radio spectrum is in many cases too flat to be explained with the traditional scenario. Instead, particle spectral indices closer to $s=1.5$ seem to be required [1].

\section{References}

[1] Aatrokoski J, Ade P, Aghanim N et al. 2011 A\&A 536 A15

[2] Dermer C D, Finke J D, Krug H and Böttcher M 2009 ApJ 692 32-46

[3] Weidinger M and Spanier F 2010 A\&A 515 A18

[4] Lindfors E J, Türler M, Valtaoja E et al. 2006 A\&A 456 895-903

[5] Böttcher M 2010 in proc. of "Fermi meets Jansky: AGN in Radio and Gamma Rays" ed Savolainen T et al. (MPIfR, Bonn, Germany) p 41 (Preprint arXiv: 1006.5048 )

[6] Poutanen J and Stern B 2010 ApJL 717 L118-L121

[7] Finke J D and Dermer C D 2010 ApJL 714 L303-L307

[8] Aleksić J, Antonelli L, Antoranz P et al. 2011 ApJL 730 L8

[9] Stamerra A, Becerra J, Bonnoli G et al. 2011 ArXiv e-prints (Preprint 1111. 0077)

[10] Valtaoja E and Teräsranta H 1996 A\&As 120491

[11] Lähteenmäki A and Valtaoja E 2003 ApJ 590 95-108

[12] León-Tavares J, Valtaoja E, Tornikoski M et al. 2011 A\&A 532 A146

[13] Jorstad S G, Marscher A P, Mattox J R et al. 2001 ApJ 556 738-748

[14] Agudo I, Jorstad S G, Marscher A P et al. 2011 ApJL 726 L13

[15] Kovalev Y Y, Aller H D, Aller M F et al. 2009 ApJL 696 L17-L21

[16] Pushkarev A B, Kovalev Y Y and Lister M L 2010 ApJL 722 L7-L11

[17] Valtaoja E, Tornikoski M, León-Tavares J et al. 2010 in proc. of "Fermi meets Jansky: AGN in Radio and Gamma Rays" ed Savolainen T et al. (MPIfR, Bonn, Germany) pp 73-76

[18] Türler M and Björnsson C I 2011 in proc. of "Beamed and Unbeamed Gamma-Rays from Galaxies" ed Wagner R, Maraschi L and Sillanpää A (JCPS proc., in press) (Preprint arXiv:1109.2518)

[19] Abdo A A, Ackermann M, Ajello M et al. 2009 ApJ 699 817-823

[20] Marscher A P and Gear W K 1985 ApJ 298114

[21] Valtaoja E, Teräsranta H, Urpo S et al. 1992 A\&A 25471

[22] Cotton W D, Wittels J J and Shapiro I I 1980 ApJL 238 L123-L128 
[23] Valtaoja E, Haarala S, Lehto H and Valtaoja L 1988 A\&A 203 1-20

[24] Hughes P A, Aller H D and Aller M F 1991 ApJ 374 57-71

[25] Gear W K, Stevens J A, Hughes D H et al. 1994 MNRAS 267167

[26] Tammi J 2008 Proc. Int. Cosmic Ray Conf. vol 2 pp 251-254

[27] Tammi J 2008 Int. J. of Modern Phys. D 17 1811-1817

[28] Virtanen J J P and Vainio R 2005 ApJ 621 313-323

[29] Tammi J and Duffy P 2009 MNRAS 393 1063-1069

[30] Weidinger M, Rüger M and Spanier F 2010 ASTRA 6 1-7 